\title{
Parametric Study of Strain Rate Effects on Nanoparticle-Reinforced Polymer Composites
}

\author{
B. Soltannia, ${ }^{1}$ I. Haji Gholami, ${ }^{2}$ S. Masajedian, ${ }^{3}$ P. Mertiny, ${ }^{1}$ D. Sameoto, ${ }^{1}$ and F. Taheri ${ }^{4}$ \\ ${ }^{1}$ Mechanical Engineering Department, University of Alberta, Edmonton, AB, Canada T6G $1 H 9$ \\ ${ }^{2}$ Mechanical Engineering Department, Sharif University of Technology, Tehran 1458889694, Iran \\ ${ }^{3}$ Civil Engineering Department, University of Alberta, Edmonton, Canada \\ ${ }^{4}$ Civil and Resource Engineering Department, Dalhousie University, Halifax, NS, Canada B3H 4R2
}

Correspondence should be addressed to F. Taheri; farid.taheri@dal.ca

Received 4 September 2015; Revised 22 October 2015; Accepted 27 October 2015

Academic Editor: Timon Rabczuk

Copyright (c) 2016 B. Soltannia et al. This is an open access article distributed under the Creative Commons Attribution License, which permits unrestricted use, distribution, and reproduction in any medium, provided the original work is properly cited.

\begin{abstract}
Crashworthiness, energy absorption capacity, and safety are important factors in the design of lightweight vehicles made of fiberreinforced polymer composite (FRP) components. The relatively recent emergence of the nanotechnology industry has presented a novel means to augment the mechanical properties of various materials. As a result, recent attempts have contemplated the use of nanoparticles to further improve the resiliency of resins, especially when resins are used for mating FRP components. Therefore, a comprehensive understanding of the response of nanoreinforced polymer composites, subjected to various rates of loading, is of paramount importance for developing reliable structures. In this paper, the effects of nanoreinforcement on the mechanical response of a commonly used epoxy resin subjected to four different strain rates, are systematically investigated. The results are then compared to those of the neat resin. To characterize the mechanical properties of the nanocomposite, a combination of the strain rate-dependent mechanical (SRDM) model of Goldberg and his coworkers and Halpin-Tsai's micromechanical approach is employed. Subsequently, a parametric study is conducted to ascertain the influences of particle type and their weight percentage. Finally, the numerical results are compared to the experimental data obtained from testing of the neat and the nanoreinforced epoxy resin.
\end{abstract}

\section{Introduction}

Nanoparticle-reinforced polymer composites (NRPs) are receiving special attention, especially for bonding applications in automotive, marine, aerospace, and oil and gas industries. NRPs are known to enhance the mechanical, electrical, thermal, and permeability properties and diffusion barrier attributes of their host polymers. Moreover, in some cases, they could also provide self-healing ability. The enactment of mechanical properties gained by inclusion of nanoparticles includes improved strength and stiffness to weight and cost ratios, improved fatigue and corrosion resistance, more controllable damage mechanism, and augmentation of the energy-absorption capacity of their host polymer. Therefore, these attributed properties render them as effective candidates for reinforcing polymers [1-11].
Nanoparticle-reinforced polymer composites (NRPs) are increasingly being used in various engineering applications, especially in the form of adhesives. Adhesives are becoming increasingly more popular for joining structural components because of the recent advancements in techniques used for toughening them. One such emerging technique has been the inclusion of an appropriate amount of inexpensive nanocarbon, namely, Graphene Nanoplatelets (GNPs), in resins/adhesives. GNPs, which have larger diameter and aspect ratio than their more commonly used nanocarbon tubes counterpart, are less expensive and are more widely available.

One of the thrusts of our current research has been to promote the use of NRPs, in form of adhesives, in automobile applications. In such applications, the NRPs become often subject to large loading rates. Unfortunately, there is a 
clear paucity in databases that convey the performance of nanoparticle-reinforced adhesives, especially when subject to high loading (or strain) rates. This issue, in turn, has impeded the greater usage of NRPs in such engineering applications. Therefore, the mechanical characterization of such nanoreinforced adhesives is vital, especially when they are subject to large loading rates.

The use of carbon nanotubes, which are essentially sheets of graphite rolled into tubes $[12,13]$, was first proposed by Iijima in 1991 and later by Iijima and Ichihashi in 1993. Even though CNTs (both single-walled and multiwalled varieties) are quite flexible in the direction perpendicular to their longitudinal axis [14], the strength and elastic modulus of MWCNTs have been estimated to be as high as $150 \mathrm{GPa}$ and $900 \mathrm{GPa}$, respectively [15]. Their superior mechanical characteristics and optimal length-to-diameter ratio have rendered CNTs as an effective reinforcing agent for resins. Nevertheless, in order to fully harness the exceptional mechanical properties of CNTs, a strong interfacial bond between the polymer matrix and CNTs must exist [16].

Since the advent of nanotechnology, nanoparticlereinforced adhesives have also been increasingly used in many other advanced applications such as naval, automotive, aerospace, and medical industries. Kim and Reneker reported that Young's modulus of a nanofiber-reinforced composite was ten times greater than that of the neat adhesive [17]. It has also been demonstrated that MWCNT particles enhance the strength and toughness of epoxies, because nanoparticles strengthen the polymeric chains of the resin and resist crack initiation and propagation by acting as bridges [18]. Qian et al. suggested that nanotubes (CNTs) could enhance the composite strength by as much as $25 \%$ [19]. It should also be noted that a nanoparticle-reinforced adhesive with the most optimum properties can only be produced if the nanoparticles are distributed uniformly and fully dispersed (exfoliated) within the polymer. This is a very challenging process, because the formation of agglomerations can act as defect regions, thus reducing the performance instead of enhancing it.

Despite the noted studies, to date, there are only a limited number of studies that have explored the mechanical properties of resins reinforced with carbon nanoparticles, especially GNPs. Moreover, the lack of such studies becomes even more noticeable when one requires information on the effect of loading/strain rates on the response of nanoparticlereinforced resins.

Therefore, in order to better understand the mechanical behavior of the nanoparticle-reinforced polymer composites under dynamic loading conditions (hence, to be able to establish and assess the safety factor of the structure hosting NRPs), one should gain a better understanding of the mechanical response of such resins under different strain rates. Several researchers have demonstrated the ratedependent mechanical behavior of neat polymers and have developed constitutive models for their characterization (see, for instance, [20-28]). Notwithstanding the abovementioned studies and those alike, there exists no unified and robust theoretical approach capable of mimicking the atomic interaction between nanoparticles and polymers. The involved challenges in constructing such a model have therefore necessitated the use of multiscale techniques to identify the macroscale mechanical behavior of nanoparticle-reinforced polymer composites. An example of such modeling approach can be found in the work of Shokrieh et al. [29]. They incorporated a strain rate-dependent continuum-based macroscale level constitutive model developed by Goldberg et al. [22-24], combined with the Halpin-Tsai micromechanical model [30], to study the loading rate effects on a CNT-reinforced polymer composite.

In this paper, using a similar approach adopted by Shokrieh et al. [29], the effects of nanoreinforcement on the mechanical response of a nanoreinforced resin, subjected to different strain rates, are modeled and systematically investigated. More specifically, neat and nanoreinforced resins are subjected to tensile loadings applied at the rates of 1.5, 15, 150, and $1500 \mathrm{~mm} / \mathrm{min}$ (strain rates of $10^{-3}, 10^{-2}, 10^{-1}$, and $1 \mathrm{~s}^{-1}$ ). Subsequently, a parametric study is conducted to ascertain the influences of various parameters (i.e., the particle type, their weight percentage) on the mechanical properties of the nanocomposite. Finally, the numerical results are compared to the experimental data obtained for the neat and the nanoreinforced epoxy resins $[31,32]$.

\section{Experimental Investigation}

2.1. Materials and Specimen Preparation. A commonly used thermoset epoxy resin (i.e., the West System 105 resin and 206 hardener (Bay City, MI)) was used as the baseline adhesive to fabricate the test specimens. This resin was selected, because it is a commonly used resin, has a relatively low cost, and is readily available.

In order to establish a cost-effective means for enhancing the mechanical properties of the resin/adhesive, different types of nanocarbon particles were utilized as the reinforcing agent. However, the uniform distribution and dispersion of nanocarbon particles in resin were found to be quite timeconsuming and challenging, hence increasing the processing cost. It should be noted that not only do the distribution and dispersion of particles directly influence adhesive's mechanical properties, but also more importantly the nanoparticle agglomeration results in significant statistical discrepancies in performance of the final product.

Three different forms of nanocarbon particles were chosen to be dispersed into the epoxy resin:

(i) Graphitized Carbon Nanofibers (CNFs) with an outer diameter of 200 to $600 \mathrm{~nm}$ and more than $99.9 \%$ purity (obtained from the US Research Nanomaterials, Inc., Houston, TX).

(ii) Graphene Nanoplatelets (GNP-M-25) with an average diameter of $25 \mu \mathrm{m}$, thickness of $6 \mathrm{~nm}$, and surface area of $100 \mathrm{~m}^{2} / \mathrm{g}$ (obtained from XG Science Ltd., Lansing, MI).

(iii) Multiwalled Carbon Nanotubes (MWCNTs) with an outer diameter of 5 to $15 \mathrm{~nm}$ and more than $95 \%$ purity (obtained from the US Research Nanomaterials, Inc., Houston, TX). 
To facilitate uniform dispersion of the nanoparticles in resin, a mechanical stirrer was used first. Each slurry of resin/nanoparticle was stirred at a speed of $2000 \mathrm{rpm}$ for $10 \mathrm{~min}$. This procedure was followed by calendering the resin/nanoparticles mix with a three-roll mill. The calendering rollers' speed was set to the machine's maximum rotational speed of 174 RPM. To maximize dispersion quality and minimize agglomerations, the calendering was performed seven times. The roller gaps were set at $20 \mu \mathrm{m}$. Note that concentrations of CNF, MWCNT, and GNP with weight percentages of $0.25 \%, 0.5 \%$, and $1 \%$ were considered. It should be noted that selection of the rollers gap size is quite important. It is postulated that when the nanoparticles are dispersed into adhesives, they are surrounded by monomer molecules. After each round, the quality of the distribution and dispersion was monitored by sampling the mixed slurry and assessing the uniformity of the dispersion with a digital microscope.

The subsequent addition of the curing agent would initiate the chemical reaction process of the resin, promoting the monomer molecules to surround the GNPs as the resin cures. In addition to the mechanical bonding, chemical bonding known as Van der Waals bond is also generated. The inclusion of nanoparticles into resin also increases resin's viscosity (thickens it). It should also be noted that, in comparison to other types of nanoparticles (e.g., CNFs or CNTs), the relatively greater aspect ratio of GNPs helps to increase the resin/nanoparticles interactions, thus forming a tougher adhesive.

Following the aforementioned procedures, the curing agent (hardener) was added to the slurry and mixed using a stirrer at a speed of $400 \mathrm{rpm}$ for 4 to 6 minutes. The mixture was then degassed under $28^{\prime \prime} \mathrm{Hg}$ vacuum for 2 to 3 minutes (the degassing time period depends on the gel time of the resin). After degassing, the mixture was poured into appropriately designed and fabricated molds and allowed to cure for at least 12 hours at room temperature. The final products in the form of dog-bone tensile coupons with dimensions as per ASTM D638 for both neat and nanoparticle-reinforced resins are shown in Figure 1 [33].

\subsection{Characterization of the Mechanical Properties of Adhe-} sives. The prepared dog-bone shaped samples were tested in tension using an Instron servo-hydraulic universal test machine equipped with $8500^{+}$electronics. To establish the stress-strain characteristics of the neat and nanoreinforced resins, the specimens were subjected to a displacementcontrolled tensile loading [33]. First, tensile tests were performed on the neat resin at room temperature, at crosshead speeds of $1.5,15$, and $150 \mathrm{~mm} / \mathrm{min}$, per ASTM D 897 (as the baseline tests for the static and quasistatic loading conditions), and at $1500 \mathrm{~mm} / \mathrm{min}$, per ASTM D 950 (as the baseline tests for the high-strain rate loading condition) $[34,35]$. Subsequently, the reinforced adhesive specimens underwent testing with the same loading rates. The gaugelength displacement of specimens tested under quasistatic loading rates $(1.5 \mathrm{~mm} / \mathrm{min})$ was recorded using an Instron extensometer (Instron Industrial Products, Grove, PA) as well as an EIR Laser Extensometer (Electronic Instrument

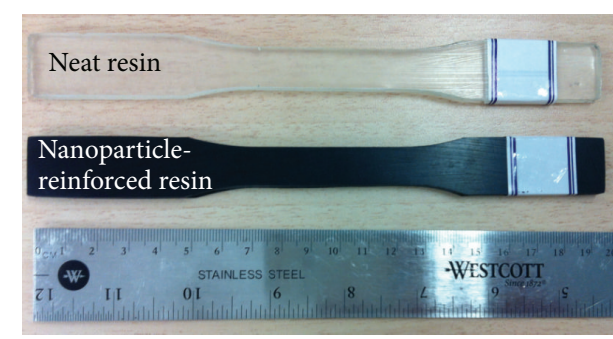

(a)

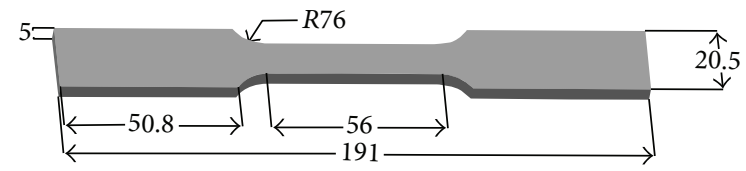

(b)

Figure 1: Representative tensile coupons of the neat and nanoparticle-reinforced epoxy resins and their dimensions (in $\mathrm{mm})$.

Research, Irwin, PA). An Instron dynamic extensometer and the laser extensometer were used to record the gaugelength displacement of the specimens tested under the quasistatic and higher loading rate experiments (i.e., 15, 150, and $1500 \mathrm{~mm} / \mathrm{min}$ ), as shown in Figure 2. Using the recorded load and gauge length displacement, the stress-strain curve of each adhesive was constructed and their elastic modulus was evaluated. The initial gauge length for all cases was $25.4 \mathrm{~mm}$.

The experimental results revealed that not only did the inclusion of nanoparticles improve the mechanical properties of the adhesive, but it also enhanced the resin's viscosity. The improved viscosity renders the adhesive suitable for use in adhesively bonded joints that oriented vertically, which are often encountered in several industrial applications, especially in marine and other applications where relatively thick bond-lines are commonly used [31, 32].

Figure 3 shows that the inclusion of the nanoparticles produced the same effect as did the increase in the strain rate. In other words, the increase in the amount of dispersed nanoparticles enhanced the stiffness of the matrix; similarly, the apparent stiffness of the composite increased as the applied strain rate increased.

\section{Problem Statement and Modeling Strategy}

The rate-dependent mechanical behavior of nanoparticlereinforced polymers has been studied by different researchers. Nonetheless, to the best of the authors' knowledge, there exists a clear lack of coherent and comprehensive parametric studies that have either theoretically or experimentally characterized the effects of nanoparticle types, their weight content, and strain rates on their response. Therefore, in this work, the macroscale rate-dependent constitutive equation of Goldberg et al. [22-24] will be modified by incorporating the Halpin-Tsai micromechanical model [30], including the inclusion of more specific shape factors, in order to assess the influence of different strain rates on the response of a polymer 


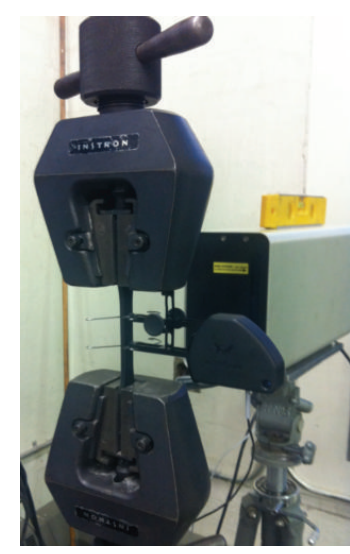

(a)

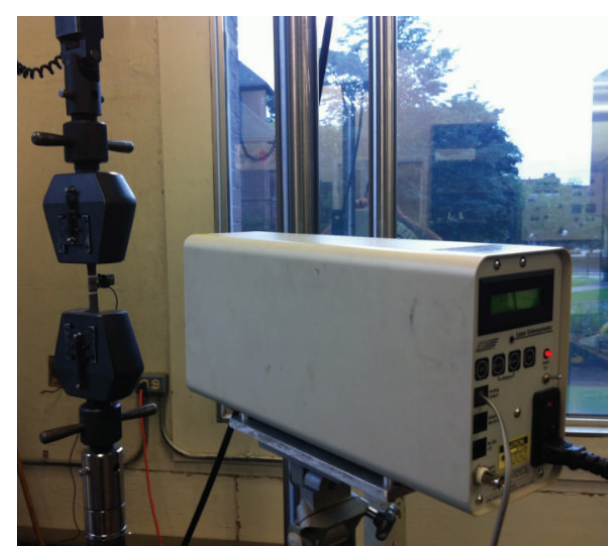

(b)

FIGURE 2: Experimental setup for the (a) static and (b) quasistatic and higher loading rate tests.

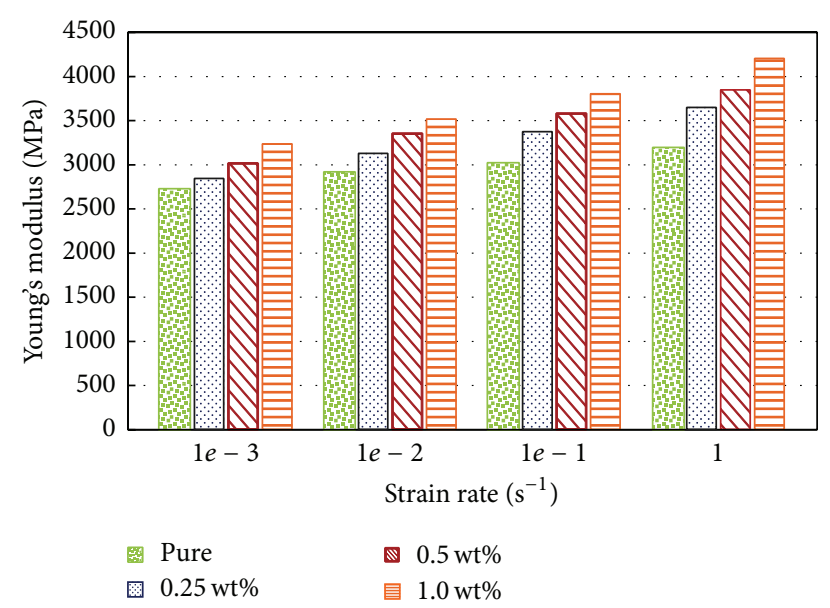

Figure 3: Influence of strain rate on the tensile elastic modulus of the epoxy resin reinforced with various amounts (wt\%) of GNPs.

reinforced by different types of nanoparticles with various weight contents. The predicted results obtained through the aforementioned superposition technique (as incorporated by Shokrieh et al. [29]) will be validated with the experimental results. At this juncture, it should be noted that one could equally adopt other micromechanical modeling techniques (such as that developed by Mori-Tanaka [4, 6], or others), in place of the Halpin-Tsai model used in this study.

\subsection{Strain Rate-Dependent Continuum-Based Constitutive} Equation of Polymers. Various researchers [21-24] have shown that the stress-strain response of nanoparticlereinforced polymer composites is dominated by the nonlinear response of its main constituent. In order to determine the viscoelastic-viscoplastic response of the polymer, the total strain $\left(\varepsilon^{T}\right)$ can be decomposed into elastic $\left(\varepsilon^{E}\right)$ and inelastic $\left(\varepsilon^{I}\right)$ components. Analogously, the inelastic strain can be calculated as the difference of the total strain and elastic strain.

Goldberg et al. [22-24] proposed a model for predicting the viscoplastic response of neat polymers, utilizing a set of state variables as an indication of the resistance of polymeric chains against flow. It should also be mentioned that polymer's mechanical properties and loading/strain rate are the two main parameters that govern the nonlinear response of the polymer. The inelastic strain components can be expressed in terms of the deviatoric stress components as follows [21-24]:

$$
\dot{\varepsilon}_{i j}^{I}=2 D_{0} \exp \left[-\frac{1}{2}\left(\frac{Z}{\sigma_{e}}\right)^{2 n}\right]\left(\frac{S_{i j}}{2 \sqrt{J_{2}}}+\alpha \delta_{i j}\right),
$$

where $\dot{\varepsilon}_{i j}^{I}$ is the inelastic strain rate tensor which can be defined as a function of deviatoric stress; $J_{2}$ is the second invariant of the deviatoric stress tensor; and $Z$ and $\alpha$ are the state variables. Moreover, $D_{0}$ and $n$ are material constants; $D_{0}$ represents the maximum inelastic strain rate; and $n$ controls the rate dependency of the material. The equivalent (effective) stress is also defined as a function of the mean stress, such that the summation of the normal stress components $\sigma_{k k}$ is three times of the mean stress, as follows:

$$
\sigma_{e}=\sqrt{3 J_{2}}+\sqrt{3} \alpha \sigma_{k k}
$$

where $\alpha$ controls the level of hydrostatic stress' effect as a state variable. The term $\dot{e}_{e}^{I}$ represents the effective deviatoric inelastic strain rate $\dot{e}_{i j}^{I}$ :

$$
\begin{gathered}
\dot{e}_{e}^{I}=\sqrt{\frac{2}{3} \dot{e}_{i j}^{I} \dot{e}_{i j}^{I}}, \\
\dot{e}_{i j}^{I}=\dot{\varepsilon}_{i j}^{I}-\frac{\dot{\varepsilon}_{k k}^{I}}{3} \delta_{i j} .
\end{gathered}
$$

The rate of change of the other two-state variables, $Z$ and $\alpha$, can be determined using the following evolution equations:

$$
\begin{aligned}
& \dot{Z}=q\left(Z_{1}-Z\right) \dot{e}_{e}^{I}, \\
& \dot{\alpha}=q\left(\alpha_{1}-\alpha\right) \dot{e}_{e}^{I} .
\end{aligned}
$$


Here, $q$ is a material constant, representing the hardening rate, which is determined through trial and error, based on the inelastic shear strain attaining a plateau, or the tensile strain corresponding to the saturation region of the stressstrain curve. $Z_{1}, \alpha_{1}$ are material constants, representing the maximum values of $Z$ and $\alpha . Z_{0}$ and $\alpha_{0}$ are the initial values of the material constants $Z$ and $\alpha . Z_{0}$ is the magnitude of the stress at the point where the shear stress-strain curve becomes nonlinear. $\alpha_{0}$ and $\alpha_{1}$ are defined using (2) and tensile stressstrain and shear stress-strain curves (the values of stress at the plateau and first nonlinearity points of the stress-strain curves, resp.).

The material constants $Z_{1}, Z_{0}, \alpha_{1}, \alpha_{0}, n$, and $D_{0}$ can be determined using the shear stress-strain and tensile or compression stress-strain curves, obtained by experiments conducted under constant strain rates on neat polymers. Empirically, it has been shown that the value of $D_{0}$, quantitatively, can be set equal to $10^{4}$ times the maximum applied total strain rate; qualitatively, it is the restricting (controlling) value of the inelastic strain rate. The values of $n, Z_{1}$ can be identified using the shear stress-strain curves constructed under various strain rates. The plateau region of the effective stress under a uniaxial tensile loading at a particular strain rate corresponds to the saturation region of the effective stress obtained under pure shear loading.

To solve the implicit Goldberg constitutive equation (1), one should utilize an appropriate numerical discretization technique. Moreover, one should ensure that the selected technique for solving the equation would be stable. For that, the four-step Runge-Kutta (R-K-4) method is adopted and implemented in this work, which is similar to the procedure that was first proposed by Tabiei and Aminjikarai [36, 37] and was also used by Shokrieh et al. [29]. The numerical representations of (1) and (5) take the following incremental forms after multiplying the rate-dependent equation by the time step $(d t)$ :

$$
\begin{aligned}
d \varepsilon_{i j}^{I} & =\left(2 D_{0} \exp \left[-\frac{1}{2}\left(\frac{Z}{\sigma_{e}}\right)^{2 n}\right]\left(\frac{S_{i j}}{2 \sqrt{J_{2}}}+\alpha \delta_{i j}\right)\right) d t, \\
d e_{i j}^{I} & =d \varepsilon_{i j}^{I}-\frac{d \varepsilon_{k k}^{I}}{3} \delta_{i j}, \\
d e_{e}^{I} & =\sqrt{\frac{2}{3} d e_{i j}^{I} d e_{i j}^{I}} \\
d Z & =q\left(Z_{1}-Z\right) d e_{e}^{I}, \\
d \alpha & =q\left(\alpha_{1}-\alpha\right) d e_{e}^{I} .
\end{aligned}
$$

As in any forward numerical method, the parameters' values from a previous step are available to be used as an input in the subsequent step. Therefore, usually a value less than the yield stress is considered as the initial guess for $\sigma_{i j}^{n}$ to start the numerical integration. Another given input parameter is the total strain rate, $\dot{\varepsilon}_{i j}^{(n+1)}$, which can be described as

$$
\varepsilon_{i j}^{(n+1)}=\varepsilon_{i j}^{(n)}+\dot{\varepsilon}_{i j}^{(n+1)} d t
$$

As the first time step of the R-K-4, values of $\sigma_{i j}=$ $\sigma_{i j}^{m(0)}, Z=Z^{m(0)}=Z_{0}$, and $\alpha=\alpha^{m(0)}=\alpha_{0}$ are considered as the input parameters (note: the superscript " $m$ " refers to the matrix). For an initial guess, the magnitude of $\sigma_{i j}^{m(0)}$ is selected such that it is less than the material's yield strength. One can then determine the values of $d \varepsilon_{i j}^{I 1}$ by substituting the values of $Z_{0}$ and $\alpha_{0}$ into (6). As a result, based on (4), (6), and (8)-(10), the first step of the R-K-4 can be represented as follows:

$$
\begin{aligned}
d \varepsilon_{i j}^{I(\text { step } 1)} & =d \varepsilon_{i j}^{I}, \\
d Z^{(\text {step } 1)} & =d Z, \\
d \alpha^{(\text {step } 1)} & =d \alpha, \\
\varepsilon_{i j}^{I} & =\varepsilon_{i j}^{I(n)}+\frac{1}{2} d \varepsilon_{i j}^{I \text { (step1) }}, \\
\{\sigma\} & =\left[C_{m}\right]\left(\{\varepsilon\}^{n+1}-\{\varepsilon\}^{I}\right), \\
Z & =Z^{m(n)}+\frac{1}{2} d Z^{(\text {step } 1)}, \\
\alpha & =\alpha^{m(n)}+\frac{1}{2} d \alpha^{(\text {step } 1)},
\end{aligned}
$$

where $\left[C_{m}\right]$ is the stiffness matrix of the polymer and the stress can be determined based on elastic and inelastic strain's constituents. The remaining steps of the $\mathrm{R}-\mathrm{K}-4$ procedure are shown in Appendix.

3.2. The Halpin-Tsai Micromechanical Model. There are different micromechanical models available for characterizing the elastic behavior of nanoparticle-reinforced polymer composites under static loading condition. The models developed by Halpin-Tsai, Mori-Tanaka, and Nielsen, to mention a few $[4-6,30]$, are some examples. The Halpin-Tsai model [4$6,30]$ used in this work can be expressed in the following form:

$$
\begin{aligned}
\frac{E_{c}}{E_{m}}=\frac{1+\xi \eta \phi}{1-\eta \phi}, & \\
& \text { where, } \eta=\left(\frac{E_{f}}{E_{m}}-1\right)\left(\frac{E_{f}}{E_{m}}+\xi\right)^{-1},
\end{aligned}
$$

where $E_{c}$ is the composite's tangential modulus, which can be determined by another equation developed by Halpin and Kardos [30], $E_{m}$ is the tangential modulus of neat polymer, determined using the predicted stress-strain curve as described in the previous section, and $E_{f}$ is the nanoparticle's modulus of elasticity. Moreover, $\phi$ is the nanoparticles volume fraction in the mixture. Depending on the nanoparticles type, different shape factors, $\xi$, would have to be used (i.e., $\xi_{\mathrm{GNP}}^{I I}=$ $2(l / t), \xi_{\mathrm{GNP}}^{\perp}=2$, and $\xi_{\mathrm{CNT}}=2(l / d)$, where $l, t$, and $d$ refer to the length, thickness, and diameter of the nanoparticles).

In the following section, the abovementioned techniques will be employed to establish the integrity of the proposed models and solution scheme. For that, first the stress-strain response of the neat resin will be predicted. That will be 
TABLE 1: Calculated rate-dependent material constants of WS-105 epoxy resin.

\begin{tabular}{|c|c|c|c|c|c|c|c|c|c|c|}
\hline Material & $\begin{array}{l}\text { Strain rate } \\
\quad\left(\mathrm{s}^{-1}\right)\end{array}$ & $\begin{array}{c}\text { Tangential } \\
\text { modulus } \\
(\mathrm{GPa})\end{array}$ & $\begin{array}{l}\text { Poisson's } \\
\text { ratio }\end{array}$ & $D_{0}\left(\mathrm{~s}^{-1}\right)$ & $n$ & $Z_{0}(\mathrm{MPa})$ & $Z_{1}(\mathrm{MPa})$ & $q$ & $\alpha_{0}$ & $\alpha_{1}$ \\
\hline WS-105 epoxy & $\begin{array}{c}1 \times 10^{-3} \\
1 \times 10^{-2} \\
1 \times 10^{-1} \\
1\end{array}$ & 3.1 & 0.3 & $5 \times 10^{6}$ & 0.745 & 200 & 1130 & 610 & 0.202 & 0.430 \\
\hline
\end{tabular}

followed by a parametric study that will assess the strainrate effects on the nanoparticle-reinforced epoxy resin. To calculate the stress-strain of nanoparticle-reinforced polymer composite, first the global stress-strain curve of the neat resin is predicted using the Goldberg model [22-24]. Then, the tensile tangent moduli of the pure polymer in appropriate strain intervals are determined, sequentially. Subsequently, the tangent moduli of the nanoparticle-reinforced resin are evaluated at each strain interval by modifying the obtained tangential moduli of the pure polymer using the Halpin-Tsai equation. As a result, the stress corresponding to each strain interval can be determined for the nanoparticle-reinforced resin. Finally, after establishing the stress values, one would be able to establish the global stress-strain curve.

\section{Results and Discussion}

In this section, we discuss the integrity and accuracy of the developed SRDM model introduced in Section 3, for predicting the tensile stress-strain response of the neat epoxy resin reinforced with various nanoparticles. For that, first by following the steps noted in Section 3 and those in Appendix, the stability of the iterative R-K-4 numerical procedure for establishing the tensile stress-strain response of the neat resin under an arbitrary strain rate is tested, with the results illustrated in Figure 4. As can be seen, three different time steps, $d t$, were selected for this task.

As seen and anticipated, smoother and more continuous curves are obtained as the value of time step is decreased, and the numerical convergence is clearly evident. To establish the rate-dependent material constants, the experimental results of the tensile tests conducted under quasistatic loading rate of $150 \mathrm{~mm} / \mathrm{min}\left(=10^{-1} \mathrm{~s}^{-1}\right)$ are used. It should be noted that, by knowing the initial gauge length (i.e., $25.4 \mathrm{~mm}$ ), one can easily convert the loading rates into strain rates. The ratedependent material properties of the neat epoxy are provided in Table 1.

Due to the nonlinear behavior of nanoparticle-reinforced polymer composites, the value of the constant linear modulus should be replaced by the tangential modulus. To establish the strain rate-dependent response of the resin reinforced with different nanoparticles and their resulting response to different strain rates, first the instantaneous tangential modulus of the neat resin under quasistatic strain rate $\left(10^{-1} \mathrm{~s}^{-1}\right)$ is established. Figure 5 illustrates the variation of the tangential tensile modulus of the neat resin.

Subsequently, having established neat resin's stress-strain response, the SRDM micromechanical model is used to

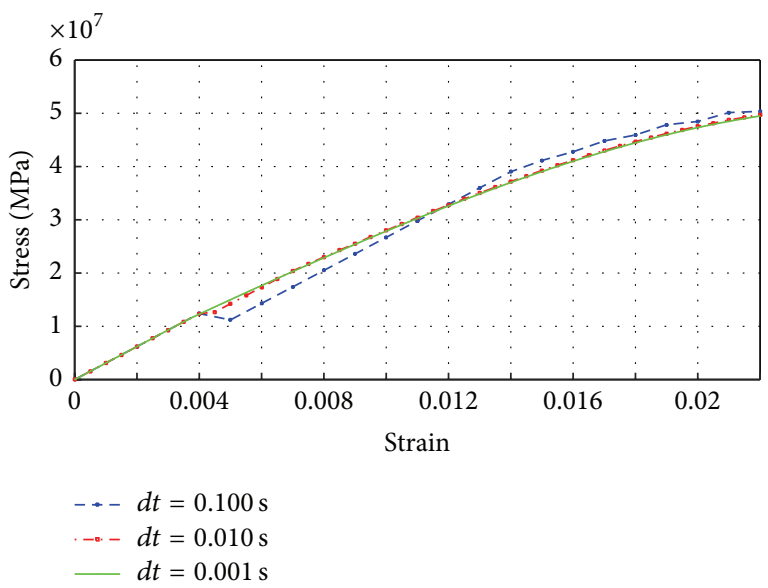

FIGURE 4: Influence of the time steps $(d t)$ on the numerical stability of the adopted iterative procedure used for predicting the stressstrain curves of the neat WS-105 epoxy resin at an arbitrary strain rate.

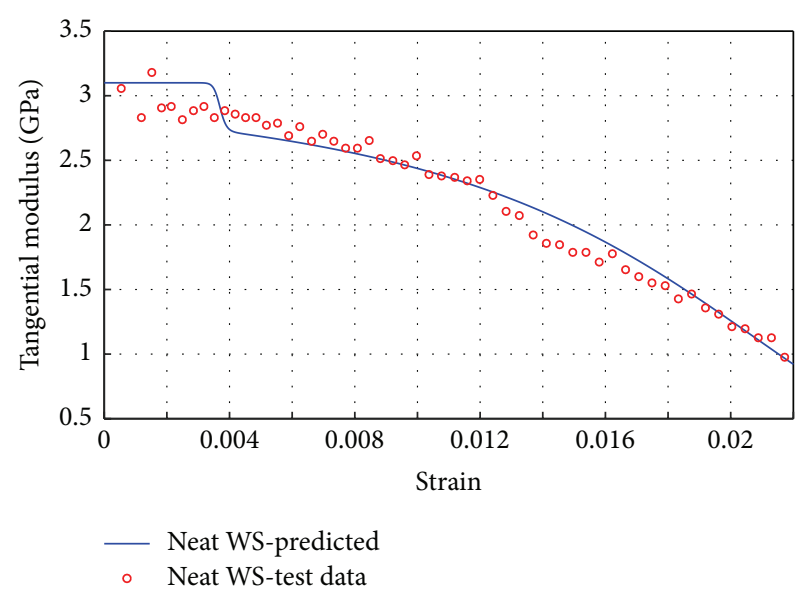

Figure 5: Variation of the tangential modulus of WS-105 neat epoxy resin, as a function of strain when the material loaded under quasistatic strain rate of $10^{-1} \mathrm{~s}^{-1}$.

establish the tensile stress-strain response of the resin reinforced with various weight contents (wt\%) of the three nanoparticle types, subjected to different strain rates. The predicted responses are then compared to the experimental results.

Figure 6 shows comparison of the predicted and experimental stress-strain curves. Figure 6(a) illustrates the variation in stress-strain response as a function of nanoparticles type under quasistatic strain rate of $10^{-3} \mathrm{~s}^{-1}$. Figure $6(\mathrm{~b})$ 

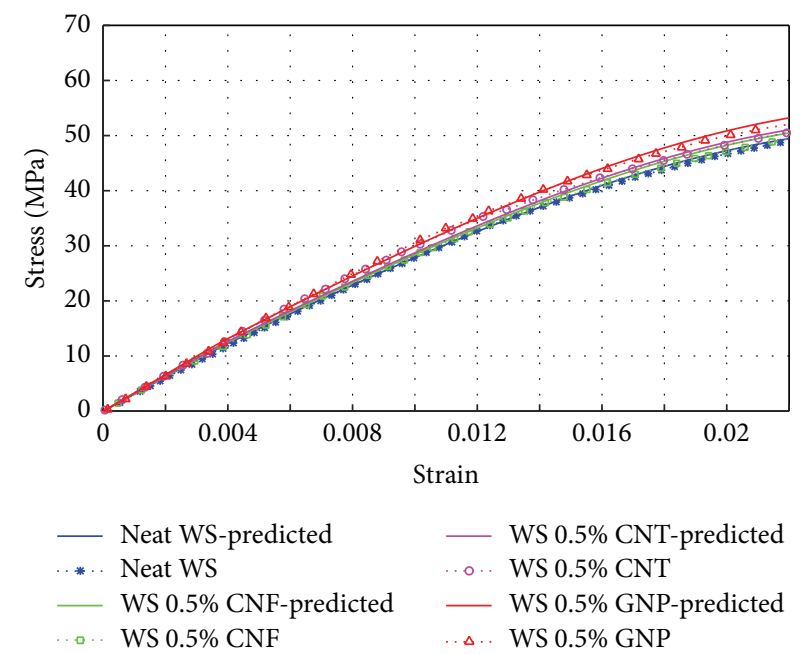

(a)

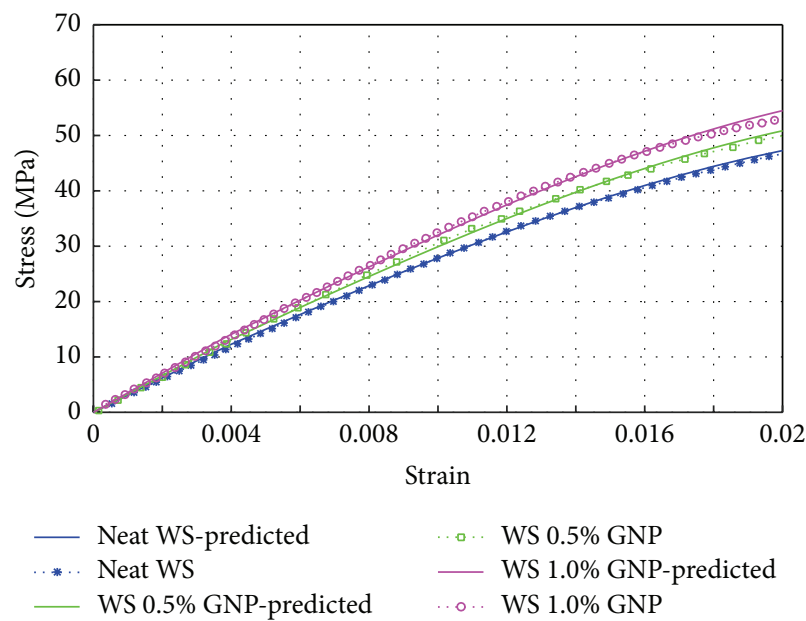

(b)

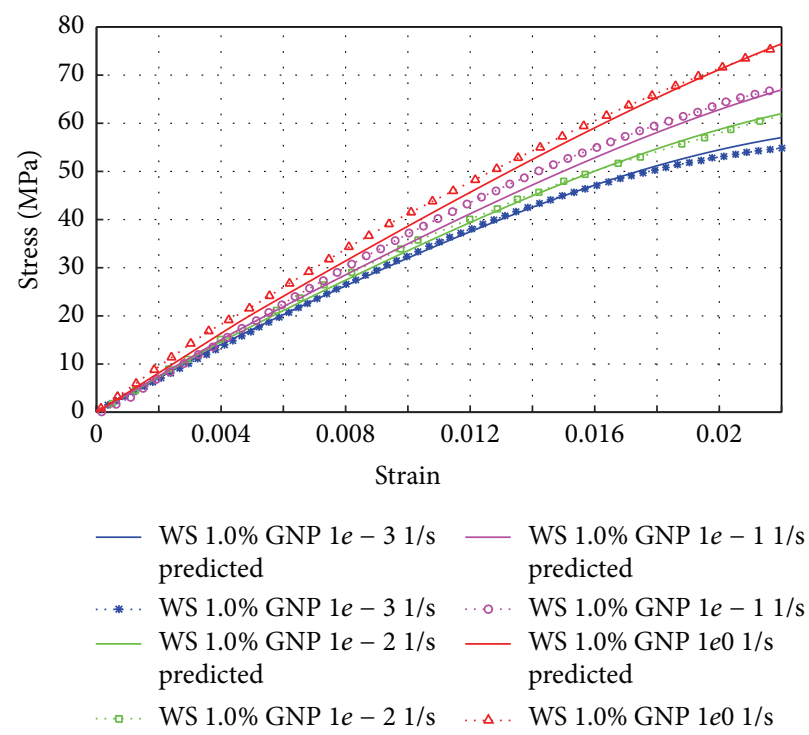

(c)

FIGURE 6: Comparison of the predicted and experimental stress-strain curves of WS-105 resins reinforced with various types of nanoparticles: (a) influence of nanoparticles type; (b) influence of GNP's weight content when the resin is subjected to a quasistatic strain rate of $10^{-3} \mathrm{~s}^{-1}$; (c) influence of strain rate on the stress-strain responses of the resin reinforced with $1 \mathrm{wt} \% \mathrm{GNP}$.

shows the influence of GNP weight content on the predicted stress-strain responses, loaded at a quasistatic strain rate of $10^{-3} \mathrm{~s}^{-1}$. Finally, Figure 6(c) illustrates the influence of the different strain rates on the predicted stress-strain responses of the resin reinforced with $1 \mathrm{wt} \% \mathrm{GNP}$. It can be seen from the results illustrated in Figure 6 that, in all cases, the predicted values are in good agreement with the experimental results.

As seen from the results shown in Figure 6(b), the inclusion of the GNPs produced the most optimum enhancement of the mechanical response of the resin. Moreover, among the weight contents considered for the inclusion of GNPs in this study, 1 wt\% was found to produce the most optimum mechanical response, regardless of the applied strain rates. It was also demonstrated that both the strength and stiffness of the nanoparticle-reinforced resin were increased as the strain rate was increased.

\section{Conclusion}

This study demonstrated that the combination of a strain rate-dependent mechanical (SRDM) model (in this case, the Goldberg et al. model) and a micromechanical model (in this case, the Halpin-Tsai model) could predict the stress-strain response of neat and nanoparticle-reinforced epoxy resins with good accuracy. The predicted results also revealed that the response of the resin was improved when it was reinforced with the relatively inexpensive type nanocarbon particles (i.e., GNPs), in comparison to the more expensive CNTs. It was also demonstrated that the increase in strain rate resulted in higher apparent strength and stiffness of nanoparticlereinforced resin. Comparatively, this increase was even more significant than the enhancement obtained by inclusion of the nanocarbon particles. Moreover, the enhancement in resin's 
stiffness was observed to be more significant compared to the gain in its strength.

As noted, inclusion of GNP into the neat resin improved the stiffness of the adhesive, resulting in enhancement of the elastic modulus by $11 \%$ and $21 \%$ for the resin reinforced $0.5 \mathrm{wt} \%$ GNPs, evaluated at the lowest and highest strain rates, respectively. Furthermore, based on the obtained results, one can conclude that the higher weight percentage (i.e., $1 \mathrm{wt} \% \mathrm{GNP}$ ) resulted in the highest stiffness. It was also observed that reinforced resin's modulus of elasticity was enhanced by $19 \%$ and $33 \%$ when the resin was reinforced with $1 \mathrm{wt} \%$ GNPs and when the material was tested under the lowest and highest loading rates, respectively.

Finally, comparison of the results obtained for the resin reinforced with $0.5 \mathrm{wt} \%$ of GNP and that reinforced with $0.5 \mathrm{wt} \%$ of $\mathrm{CNF}$, subjected to the quasistatic strain rate, revealed that inclusion of the GNP produced a greater average ultimate tensile strength and modulus of elasticity than inclusion of CNF could.

\section{Appendix}

\section{Details of the Runge-Kutta Procedure}

The second step of the R-K-4 using the results of the first step takes the following form:

$$
\begin{aligned}
d \varepsilon_{i j}^{I(\text { step2) }} & =d \varepsilon_{i j}^{I}, \\
d Z^{(\text {step2) }} & =d Z, \\
d \alpha^{(\text {step2) }} & =d \alpha, \\
\varepsilon_{i j}^{I} & =\varepsilon_{i j}^{I(n)}+\frac{1}{2} d \varepsilon_{i j}^{I \text { step2) }} \\
\{\sigma\} & =\left[C_{m}\right]\left(\{\varepsilon\}^{n+1}-\{\varepsilon\}^{I}\right), \\
Z & =Z^{m(n)}+\frac{1}{2} d Z^{(\text {step } 2)}, \\
\alpha & =\alpha^{m(n)}+\frac{1}{2} d \alpha^{(\text {step } 2)} .
\end{aligned}
$$

And the 3rd step can be obtained using the results of the 2nd step:

$$
\begin{aligned}
d \varepsilon_{i j}^{I(\text { step3) }} & =d \varepsilon_{i j}^{I}, \\
d Z^{(\text {step3) }} & =d Z, \\
d \alpha^{(\text {step3) }} & =d \alpha, \\
\varepsilon_{i j}^{I} & =\varepsilon_{i j}^{I(n)}+\frac{1}{2} d \varepsilon_{i j}^{I(\text { step} 3)}, \\
\{\sigma\} & =\left[C_{m}\right]\left(\{\varepsilon\}^{n+1}-\{\varepsilon\}^{I}\right), \\
Z & =Z^{m(n)}+\frac{1}{2} d Z^{(\text {step } 3)}, \\
\alpha & =\alpha^{m(n)}+\frac{1}{2} d \alpha^{(\text {step } 3)} .
\end{aligned}
$$

Similar to the pervious steps, results of the third step are used in the final step:

$$
\begin{aligned}
& d \varepsilon_{i j}^{I(\text { step4) }}=d \varepsilon_{i j}^{I}, \\
& d Z^{(\text {step4) }}=d Z \\
& d \alpha^{(\text {step4) }}=d \alpha .
\end{aligned}
$$

Then, the magnitude of stress and inelastic strain in the next time step can be calculated through the final step of the Runge-Kutta method as follows:

$$
\begin{aligned}
\varepsilon_{i j}^{I(n+1)}= & \varepsilon_{i j}^{I(n)}+\frac{1}{6} d \varepsilon_{i j}^{I(\text { step } 1)}+\frac{1}{3} d \varepsilon_{i j}^{I(\text { step } 2)} \\
& +\frac{1}{3} d \varepsilon_{i j}^{I(\text { step} 3)}+\frac{1}{6} d \varepsilon_{i j}^{I(\text { step } 4)}, \\
\{\sigma\}^{m(n+1)}= & {\left[C_{m}\right]\left(\{\varepsilon\}^{n+1}-\{\varepsilon\}^{I(n+1)}\right), } \\
Z^{m(n+1)}= & Z^{m(n)}+\frac{1}{6} d Z^{(\text {step } 1)}+\frac{1}{3} d Z^{(\text {step } 2)} \\
& +\frac{1}{3} d Z^{(\text {step } 3)}+\frac{1}{6} d Z^{(\text {step } 4)}, \\
\alpha^{m(n+1)}= & \alpha^{m(n)}+\frac{1}{6} d \alpha^{(\text {step } 1)}+\frac{1}{3} d \alpha^{(\text {step } 2)} \\
& +\frac{1}{3} d \alpha^{(\text {step } 3)}+\frac{1}{6} d \alpha^{(\text {step } 4)} .
\end{aligned}
$$

Finally, the tensile stress-strain curve of the neat polymer under an arbitrary strain rate can be obtained accordingly.

\section{Conflict of Interests}

The authors declare that there is no conflict of interests regarding the publication of this paper.

\section{Acknowledgments}

The Natural Science and Engineering Research Council of Canada's financial support for this research work is gratefully acknowledged and appreciated.

\section{References}

[1] M. R. Loos and K. Schulte, "Is it worth the effort to reinforce polymers with carbon nanotubes?" Macromolecular Theory and Simulations, vol. 20, no. 5, pp. 350-362, 2011.

[2] O. Breuer and U. Sundararaj, "Big returns from small fibers: a review of polymer/carbon nanotube composites," Polymer Composites, vol. 25, no. 6, pp. 630-645, 2004.

[3] G. Choudalakis and A. D. Gotsis, "Permeability of polymer/clay nanocomposites: a review," European Polymer Journal, vol. 45, no. 4, pp. 967-984, 2009.

[4] J.-J. Luo and I. M. Daniel, "Characterization and modeling of mechanical behavior of polymer/clay nanocomposites," Composites Science and Technology, vol. 63, no. 11, pp. 1607-1616, 2003. 
[5] P.-G. Ren, D.-X. Yan, T. Chen, B.-Q. Zeng, and Z.-M. Li, "Improved properties of highly oriented graphene/polymer nanocomposites," Journal of Applied Polymer Science, vol. 121, no. 6 , pp. 3167-3174, 2011.

[6] T. D. Fornes and D. R. Paul, "Modeling properties of nylon 6/clay nanocomposites using composite theories," Polymer, vol. 44, no. 17, pp. 4993-5013, 2003.

[7] D. Datashvili and H. Baier, "Membranes and thin shells for space reflectors," in Proceedings of the Earth \& Space, pp. 1-8, Chicago, Ill, USA, 2006.

[8] J.-K. Lee, "Prediction of thermal conductivity of composites with spherical fillers by successive embedding," Archive of Applied Mechanics, vol. 77, no. 7, pp. 453-460, 2007.

[9] M. A. Rafiee, J. Rafiee, Z. Wang, H. Song, Z.-Z. Yu, and N. Koratkar, "Enhanced mechanical properties of nanocomposites at low graphene content," ACS Nano, vol. 3, no. 12, pp. 38843890, 2009.

[10] J. Rotrekl, L. Matějka, L. Kaprálková, A. Zhigunov, J. Hromádková, and I. Kelnar, "Epoxy/PCL nanocomposites: effect of layered silicate on structure and behavior," Express Polymer Letters, vol. 6, no. 12, pp. 975-986, 2012.

[11] S. Shadlou, B. Ahmadi-Moghadam, and F. Taheri, "Nanoenhanced adhesives," Reviews of Adhesion and Adhesives, vol. 2, no. 3, pp. 371-412, 2014.

[12] S. Iijima, "Helical microtubules of graphitic carbon," Nature, vol. 354, no. 6348, pp. 56-58, 1991.

[13] S. Iijima and T. Ichihashi, "Single-shell carbon nanotubes of 1nm diameter," Nature, vol. 363, no. 6430, pp. 603-605, 1993.

[14] R. Saito, G. Dresselhaus, and M. S. Dresselhaus, Physical Properties of Carbon Nanotubes, Imperial College Press, London, UK, 1998.

[15] B. G. Demczyk, Y. M. Wang, J. Cumings et al., "Direct mechanical measurement of the tensile strength and elastic modulus of multiwalled carbon nanotubes," Materials Science and Engineering: A, vol. 334, no. 1-2, pp. 173-178, 2002.

[16] M. Alexandre and P. Dubois, "Polymer-layered silicate nanocomposites: preparation, properties and uses of a new class of materials," Materials Science and Engineering R: Reports, vol. 28, no. 1-2, pp. 1-63, 2000.

[17] J.-S. Kim and D. H. Reneker, "Mechanical properties of composites using ultrafine electrospun fibers," Polymer Composites, vol. 20, no. 1, pp. 124-131, 1999.

[18] V. K. Srivastava and P. J. Hogg, "Moisture effects on the toughness, mode-I and mode-II of particles filled quasi-isotropic glass-fibre reinforced polyester resin composites," Journal of Materials Science, vol. 33, no. 5, pp. 1129-1136, 1998.

[19] D. Qian, E. C. Dickey, R. Andrews, and T. Rantell, "Load transfer and deformation mechanisms in carbon nanotube-polystyrene composites," Applied Physics Letters, vol. 76, no. 20, pp. 2868$2870,2000$.

[20] R. A. Schapery, "Nonlinear viscoelastic and viscoplastic constitutive equations based on thermodynamics," Mechanics TimeDependent Materials, vol. 1, no. 2, pp. 209-240, 1997.

[21] A. Plaseied and A. Fatemi, "Deformation response and constitutive modeling of vinyl ester polymer including strain rate and temperature effects," Journal of Materials Science, vol. 43, no. 4, pp. 1191-1199, 2008.

[22] R. K. Goldberg, G. D. Roberts, and A. Gilat, "Implementation of an associative flow rule including hydrostatic stress effects into the high-strain rate deformation analysis of polymer matrix composites," Journal of Aerospace Engineering, vol. 18, no. 1, pp. 18-27, 2005.
[23] R. K. Goldberg and D. C. Stouffer, "Strain rate dependent analysis of a polymer matrix composite utilizing a micromechanics approach," Journal of Composite Materials, vol. 36, no. 7, pp. 773793, 2002.

[24] A. Gilat, R. K. Goldberg, and G. D. Roberts, "Strain rate sensitivity of epoxy resin in tensile and shear loading," Journal of Aerospace Engineering, vol. 20, no. 2, pp. 75-89, 2007.

[25] O. A. Hasan and M. C. Boyce, "Constitutive model for the nonlinear viscoelastic viscoplastic behavior of glassy polymers," Polymer Engineering and Science, vol. 35, no. 4, pp. 331-344, 1995.

[26] S. P. Bao and S. C. Tjong, "Mechanical behaviors of polypropylene/carbon nanotube nanocomposites: the effects of loading rate and temperature," Materials Science and Engineering A, vol. 485, no. 1-2, pp. 508-516, 2008.

[27] J. Ingram, Y. Zhou, S. Jeelani, T. Lacy, and M. F. Horstemeyer, "Effect of strain rate on tensile behavior of polypropylene and carbon nanofiber filled polypropylene," Materials Science and Engineering: A, vol. 489, no. 1-2, pp. 99-106, 2008.

[28] S. Peter and E. Woldesenbet, "Nanoclay syntactic foam composites-high strain rate properties," Materials Science and Engineering A, vol. 494, no. 1-2, pp. 179-187, 2008.

[29] M. M. Shokrieh, R. Mosalmani, and M. J. Omidi, "Strain rate dependent micromechanical modeling of reinforced polymers with carbon nanotubes," Journal of Composite Materials, vol. 48, no. 27, pp. 3381-3393, 2014.

[30] J. C. Halpin and J. L. Kardos, “The Halpin-Tsai equations: a review," Polymer Engineering and Science, vol. 16, no. 5, pp. 344352, 1976

[31] B. Soltannia and F. Taheri, "Static, quasi-static and high loading rate effects on graphene nano-reinforced adhesively bonded single-lap joints," International Journal of Composite Materials, vol. 3, pp. 181-190, 2013.

[32] B. Soltannia and F. Taheri, "Influence of nano-reinforcement on the mechanical behavior of adhesively bonded single-lap joints subjected to static, quasi-static, and impact loading," Journal of Adhesion Science and Technology, vol. 29, no. 5, pp. 424-442, 2015.

[33] ASTM International, "Standard test method for tensile properties of plastics," ASTM D638, ASTM International, West Conshohocken, Pa, USA, 2012.

[34] ASTM International, "Test method for tensile properties of adhesive bonds," ASTM D897, ASTM International, West Conshohocken, Pa, USA, 2012.

[35] ASTM International, "Test method for impact strength of adhesive bonds," ASTM D950, ASTM International, West Conshohocken, Pa, USA, 2012.

[36] A. Tabiei and I. Ivanov, "Micro-mechanical model with strainrate dependency and damage for impact simulation of woven fabric composites," Mechanics of Advanced Materials and Structures, vol. 14, no. 5, pp. 365-377, 2007.

[37] A. Tabiei and S. B. Aminjikarai, "A strain-rate dependent micro-mechanical model with progressive post-failure behavior for predicting impact response of unidirectional composite laminates," Composite Structures, vol. 88, no. 1, pp. 65-82, 2009. 

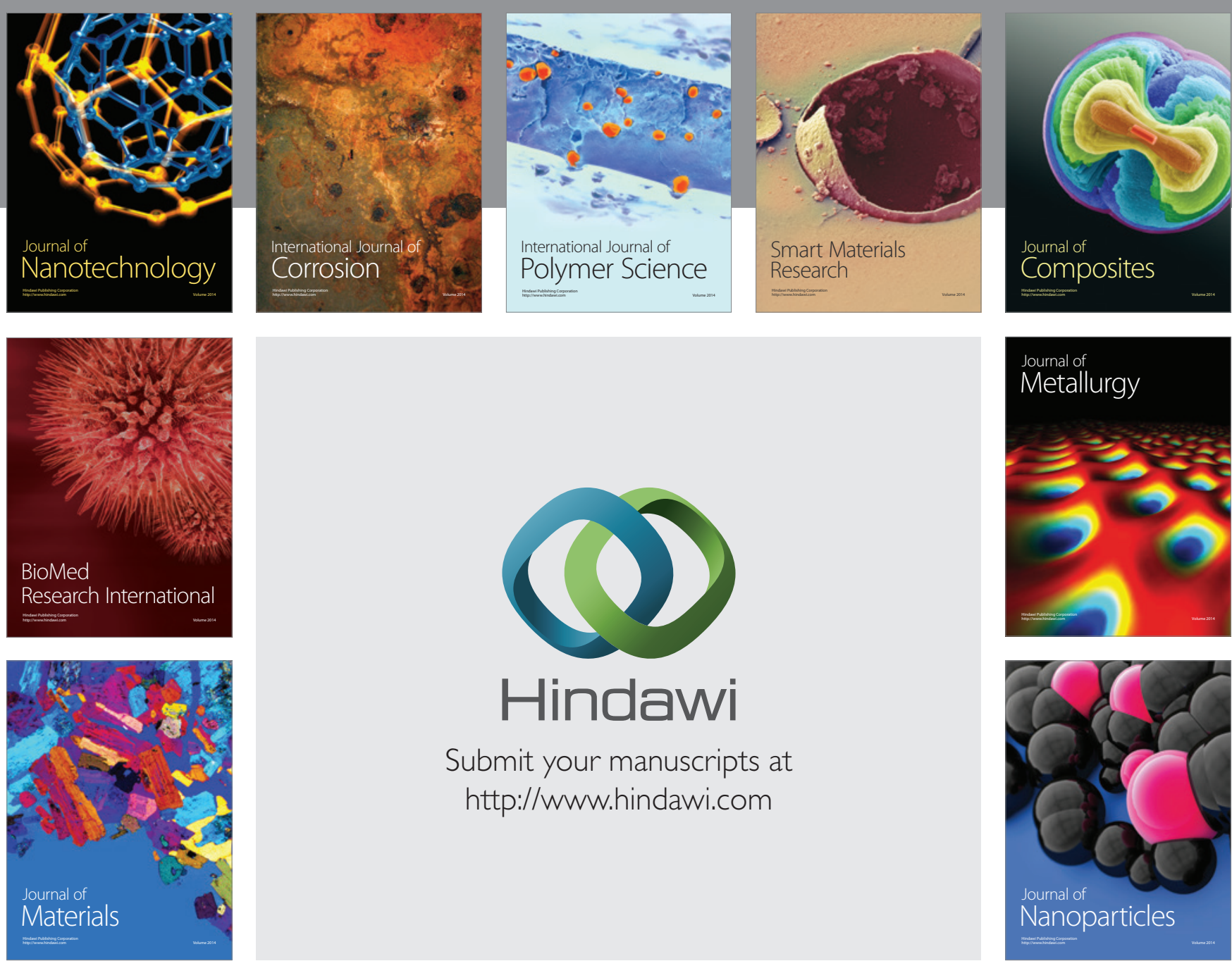

\section{Hindawi}

Submit your manuscripts at

http://www.hindawi.com

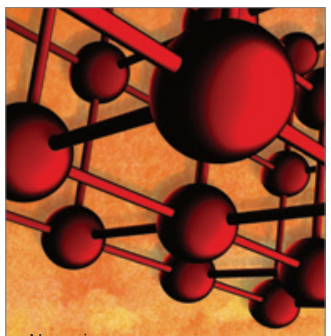

Materials Science and Engineering
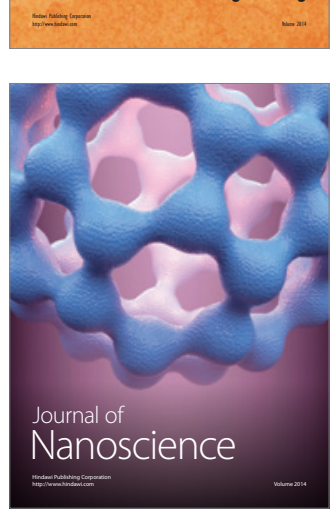
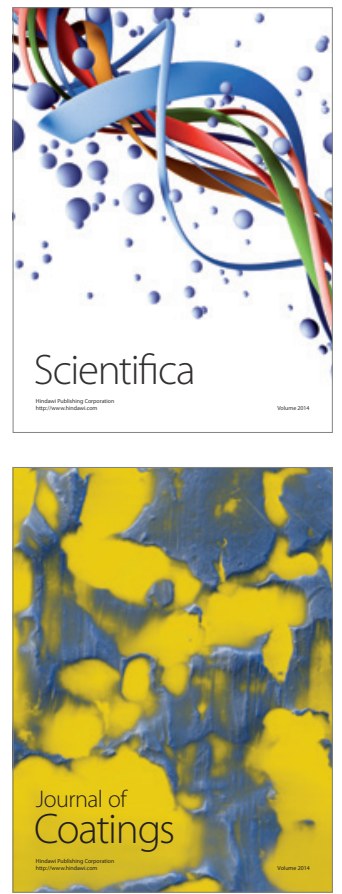
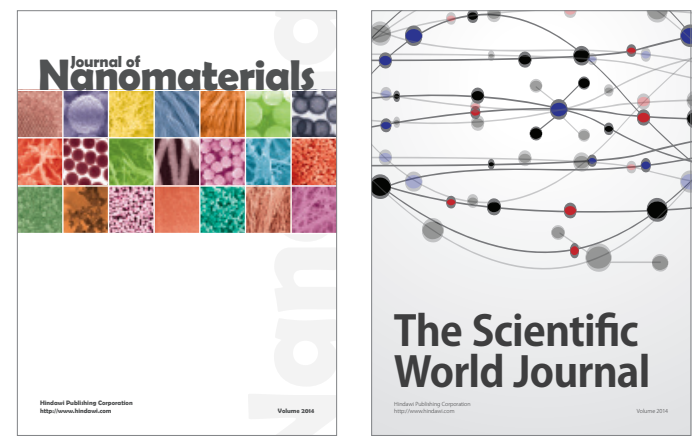

The Scientific World Journal
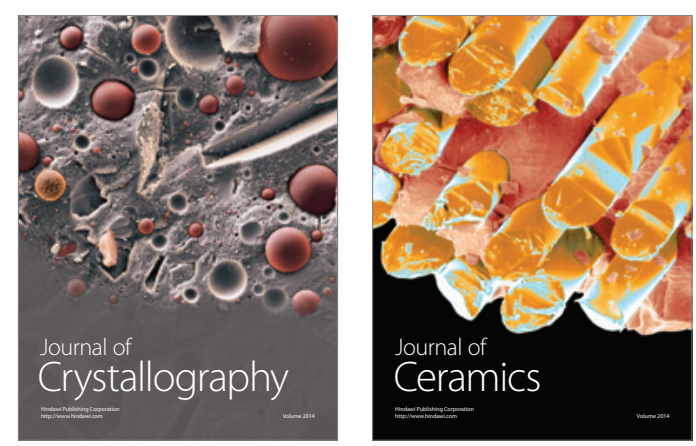
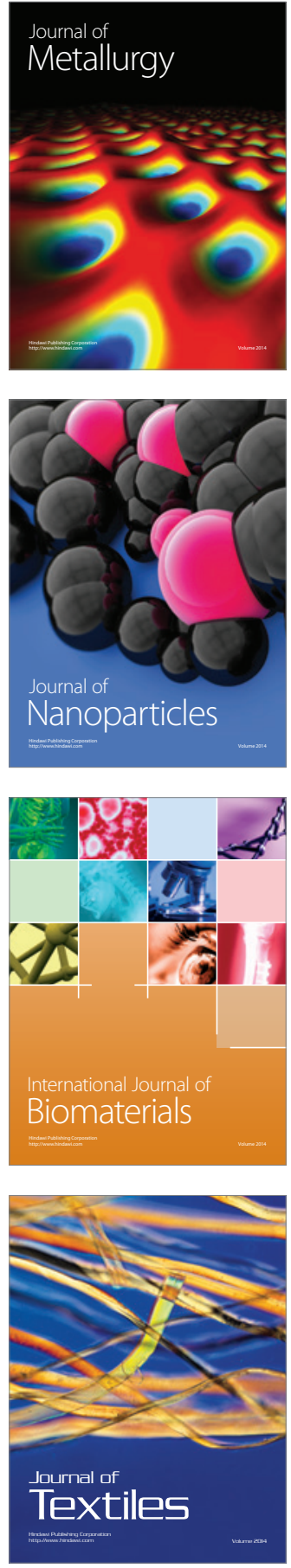